\title{
MANUTENÇÃO DE FOLHAS ATIVAS EM BANANEIRA-'NANICÃO’ POR MEIO DO MANEJO DAS ADUBAÇÕES NITROGENADA E POTÁSSICA E DA IRRIGAÇÃO'
}

\author{
LUIZ ANTONIO JUNQUEIRA TEIXEIRA², CARLOS RUGGIERO ${ }^{3}$, WILLIAM NATALE ${ }^{3}$
}

\begin{abstract}
RESUMO - Realizou-se um experimento de campo, em Jaboticabal-SP, com o objetivo de estudar a resposta da bananeira (Musa AAA subgrupo Cavendish)-'Nanicão' à adubação nitrogenada e potássica, sob irrigação e sequeiro, durante duas safras. Empregou-se o delineamento experimental de blocos ao acaso, com os tratamentos em parcelas subdivididas, sendo as parcelas principais constituídas por dois regimes hídricos: irrigado (microaspersão) e sequeiro, e as subparcelas, pelas combinações de quatro doses de $\mathrm{N}(0 ; 200 ; 400$ e $\left.800 \mathrm{~kg} \mathrm{ha}^{-1} \mathrm{de} \mathrm{N}\right)$ e quatro de $\mathrm{K}\left(0 ; 300 ; 600\right.$ e $\left.900 \mathrm{~kg} \mathrm{ha}^{-1} \mathrm{de} \mathrm{K}_{2} \mathrm{O}\right)$. O bananal foi cultivado de acordo com as recomendações atuais, tomando-se cuidados especiais com o controle preventivo de sigatoca-amarela e com o manejo da irrigação. Por meio da análise do número de folhas ativas ( $>50 \%$ da área verde) nas épocas da emissão da inflorescência (NFE) e da colheita (NFC), do índice de durabilidade foliar (IDF=NFC,NFE'100) e dos teores de N e K na folha-índice, avaliaram-se os efeitos da irrigação e da aplicação de doses crescentes de $\mathrm{N}$ e K sobre as condições das folhas. Nos dois ciclos de cultivo, houve efeito da adubação potássica e da irrigação sobre o estado das folhas $(p<0,05)$. O NFC sob irrigação $(7,2)$ foi maior do que sob sequeiro $(3,8)$. Sob sequeiro, o IDF aumentou linearmente com as doses crescentes de K. Na segunda safra, estimou-se que razões entre os teores foliares de K/N em torno de 1,6 (sequeiro) e 1,4 (irrigado) determinaram máxima durabilidade foliar $\left(\operatorname{IDF}_{\text {sequeiro }}=49 \%\right.$; $\left.\operatorname{IDF}_{\text {irrigado }}=68 \%\right)$. A irrigação e o manejo correto da adubação (evitar excesso de $\mathrm{N}$ em relação ao $\mathrm{K}$ ) demonstraram ser ferramentas eficientes para aumentar a longevidade das folhas na cultura da bananeira.
\end{abstract}

Termos para indexação: banana, nitrogênio, potássio, Musa spp., senescência

\section{IMPROVING LIFE SPAN OF THE BANANA LEAVES THROUGH NITROGEN AND POTASSIUM FERTILIZATION AND IRRIGATION}

\begin{abstract}
A field experiment was carried out in São Paulo State, Brazil, with the objective of investigating the response of 'Nanicão' banana (Musa AAA Cavendish subgroup) to nitrogen and potassium fertilization, under irrigated and non-irrigated conditions, during two crop seasons. A split-plot design was used with irrigation (micro-sprinkler) and no irrigation applied to main plots and a combination of four rates of $\mathrm{N}\left(0,200,400\right.$ and $\left.800 \mathrm{~kg} \mathrm{~N} \mathrm{ha}^{-1}\right)$ and $\mathrm{K}\left(0,300,600\right.$ and $\left.900 \mathrm{~kg} \mathrm{~K}_{2} \mathrm{O} \mathrm{ha}{ }^{-1}\right)$ as the sub-plots treatments. The area was managed according to regional recommendations; special attention was paid to disease control and irrigation management. The effects of treatments on life span of leaves were assessed counting leaves at flowering (NFE) and at bunch harvest (NFC), calculating leaf longevity index (IDF= NFC, $\left.\mathrm{NFE}^{\prime} 100\right)$ and studying $\mathrm{N}$ and $\mathrm{K}$ leaf contents. Irrigation and $\mathrm{K}$ fertilization caused a significant increase in leaf longevity $(p<0.05)$. The NFC with irrigation (7.2) was greater than in non-irrigated plots (3.8). In the first ratoon crop, the $\mathrm{K} / \mathrm{N}$ ratio that provided greatest leaf longevity was 1.6 for non-irrigated plants $\left(\mathrm{IDF}_{\text {no-iri }}=49 \%\right)$ and 1.4 under irrigation $\left(\operatorname{IDF}_{\text {irri }}=68 \%\right.$ ). Irrigation and accurate fertilization (avoidance of low $\mathrm{K} / \mathrm{N}$ ratio) showed to be useful for increasing life span of banana leaves.
\end{abstract}

Index terms: banana, Musa spp., senescence

\section{INTRODUÇÃO}

A bananicultura encontra-se presente em quase todo o País, apresentando significativa diversidade, especialmente em relação às variedades e padrão tecnológico das explorações. Segundo Souza \& Torres (1997), a área ocupada para a produção de banana é a segunda maior entre as destinadas às frutas para consumo in natura.

A manutenção de área foliar ativa por mais tempo é uma luta constante para os produtores de banana, sendo que a primeira preocupação é com o controle de doenças foliares (Sigatoka, principalmente). Robinson et al. (1992) obtiveram rendimento máximo de frutos em bananeira 'Williams' com a manutenção de oito folhas durante o período entre emissão da inflorescência e colheita; com quatro folhas, a produção de frutos comercializáveis foi afetada tanto em quantidade como em qualidade. Na prática, alguns bananicultores afirmam que deve ser mantido até a colheita, no mínimo, um número igual de folhas ativas e de pencas.

Em relação à fertilidade do solo, há relatos de que o suprimento adequado de $\mathrm{K}$ seja fundamental para a preservação da área foliar (Murray, 1960; Hasselo, 1961; Lahav, 1972; Lahav, 1995). Outrossim, segundo Delvaux (1995), a manutenção do 
equilíbrio de cátions e de adequados níveis de potássio e nitrogênio disponíveis no solo são as condições químicas mais importantes para o cultivo de bananeiras visando a altos rendimentos.

O objetivo deste trabalho foi estudar os efeitos da adubação nitrogenada e potássica, em condições de sequeiro e sob irrigação, sobre a durabilidade das folhas de bananeira no período entre a emissão da inflorescência e a colheita dos cachos.

\section{MATERIAL E MÉTODOS}

O experimento foi realizado em Jaboticabal-SP, cuja altitude é de aproximadamente $575 \mathrm{~m}$ e latitude $21^{\circ} 15^{\prime} \mathrm{S}$. O clima é mesotérmico de inverno seco (Cwa - Classificação de Koeppen), e o solo classificado como Latossolo Vermelho Eutroférrico típico. Valores médios de alguns atributos do solo, em amostragem realizada na época da instalação do experimento, encontram-se na Tabela 1. Informações meteorológicas coletadas no período de condução do experimento são apresentadas na Figura 1.

O delineamento experimental foi o de blocos ao acaso, com duas repetições e parcelas subdivididas. As parcelas principais foram constituídas por dois regimes hídricos: irrigado (microaspersão) e sequeiro. Nas subparcelas, os tratamentos, em arranjo fatorial, consistiram nas combinações de quatro doses de nitrogênio $\left(0 ; 200 ; 400\right.$ e $800 \mathrm{~kg} \mathrm{ha}^{-1}$ de $\left.\mathrm{N}\right)$ e quatro de potássio $\left(0 ; 300 ; 600\right.$ e $900 \mathrm{~kg} \mathrm{ha}^{-1}$ de $\left.\mathrm{K}_{2} \mathrm{O}\right)$. As unidades experimentais (subparcelas) foram compostas por vinte plantas $\left(104 \mathrm{~m}^{2}\right)$, sendo seis consideradas para as avaliações (plantas úteis). As doses de adubo (nitrato de amônio e cloreto de potássio, como fontes de $\mathrm{N}$ e $\mathrm{K}$, respectivamente) foram parceladas em quatro vezes durante a estação das chuvas. Os fertilizantes foram sempre aplicados na superfície do solo sem incorporação. No estabelecimento do bananal, foi aplicado fósforo (170 $\mathrm{kg} \mathrm{ha}^{-1} \mathrm{de}$ $\mathrm{P}_{2} \mathrm{O}_{5}$ ) sob a forma de termofosfato.

O bananal foi implantado empregando-se mudas micropropagadas in vitro da cultivar Nanicão (Musa AAA subgrupo Cavendish), em outubro de 1997. Sua condução foi de acordo com as recomendações descritas por Moreira (1999). Fezse o controle de doenças, especialmente sigatoca-amarela, e pragas (nematóides e broca) de forma preventiva, por meio da aplicação de defensivos indicados para a cultura em épocas predeterminadas.

Os teores de $\mathrm{N}$ e $\mathrm{K}$ foliar foram estimados por meio de amostragem realizada conforme as recomendações de MartinPrével (1984). As amostras de tecido foliar foram processadas e analisadas quanto aos teores de $\mathrm{N}$ e $\mathrm{K}$ de acordo com Bataglia et al. (1983). Na época da emissão da inflorescência, contaram-se as folhas ativas, ou seja, aquelas que apresentavam mais da metade do limbo verde (NFE). Na colheita do cacho, determinouse novamente o número de folhas ativas (NFC).

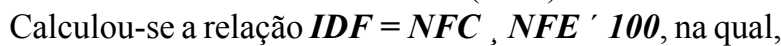
$I D F$ é o índice de durabilidade foliar (\%), $N F C$, o número de folhas ativas na época da colheita do cacho e $\boldsymbol{N F E}$, o número de folhas ativas na época da emissão da inflorescência.

Os efeitos dos tratamentos aplicados às parcelas principais (sequeiro e irrigação) foram avaliados empregando-se o teste F. Para as situações nas quais se detectaram efeitos significativos dos tratamentos de adubação, foram ajustadas equações de regressão relacionando doses de potássio e nitrogênio com as variáveis respostas, para condições de sequeiro e irrigação. Nas análises de variância, empregou-se o módulo GLM (General Linear Models) do SAS, segundo Freund \& Litttell (1981); os parâmetros dos modelos de regressão foram estimados por máxima verossimilhança, empregando-se o módulo MIXED (Mixed Models), conforme Littell et al. (1996).

\section{RESULTADOS E DISCUSSÃO}

Nos dois ciclos de cultivo, houve efeito $(p<0,05)$ da adubação potássica e da irrigação sobre a durabilidade das folhas e número de folhas existentes na época da colheita (Tabela 2), não obstante a realização de um eficiente controle preventivo de sigatoca-amarela. Apesar de a irrigação possibilitar maior absorção de $\mathrm{Ne} \mathrm{K}$, não foi detectado efeito significativo $(p>0,05)$ do regime hídrico sobre os teores foliares de $\mathrm{N}$ ou $\mathrm{K}$. Provavelmente, ao incrementar o crescimento das plantas, a quantidade de nutrientes no tecido foliar foi diluída num volume maior. Os maiores efeitos sobre a concentração de $\mathrm{N}$ e $\mathrm{K}$ nas folhas foram decorrentes da aplicação dos fertilizantes.

Na Tabela 3, é apresentado o efeito da irrigação (médias de todos os tratamentos de adubação com $\mathrm{N}$ e K) sobre as variáveis relacionadas ao estado das folhas. Ressalta-se que, com irrigação, foi possível atingir o período de colheita com quase o dobro de folhas do que sob a condição de sequeiro, pois as folhas existentes na época da emissão da inflorescência tiveram sua durabilidade favorecida pela maior disponibilidade de água. A irrigação possibilitou que mais da metade das folhas existentes na época da emissão da inflorescência se mantivessem ativas até o momento da colheita (IDF $>50 \%$ ). Sob sequeiro, o IDF foi aproximadamente $30 \%$ nos dois ciclos de cultivo. Pelos resultados, infere-se que o estresse hídrico acelerou a senescência foliar, freqüentemente resultando em redução irreversível da área fotossinteticamente ativa. Boyer (1976) considerou que essa associação entre perda de área foliar e diminuição na fotossíntese representa um grande potencial de diminuição na produção de fotossintetizados, com reflexos sobre a produção. $\mathrm{O}$ efeito da irrigação sobre a durabilidade foliar, observado no presente trabalho, foi semelhante ao determinado por Manica et al. (1975) para 'Nanicão'. Esses autores constataram que a manutenção de um número maior de folhas até a colheita foi favorecida pela irrigação, observando que, na época da colheita, as plantas sob sequeiro apresentavam, em média, 3,7 folhas, enquanto as plantas irrigadas estavam com 6,4 folhas. A irrigação em bananeiras 'Robusta', segundo Holder \& Gumbs (1983), também determinou um incremento significativo na durabilidade das folhas.

$\mathrm{Na}$ Figura 2, tem-se a relação entre o índice de durabilidade foliar e a aplicação de potássio. Em sequeiro, o IDF aumentou linearmente com as doses crescentes de $\mathrm{K}$ nas duas safras. Chama a atenção, a obtenção de resposta à adubação potássica num solo com teor médio de $\mathrm{K}$ trocável superior a 3,0 $\mathrm{mmol}_{\mathrm{c}} \mathrm{dm}^{-3}$. Observa-se que essa resposta foi obtida somente em sequeiro, indicando que a irrigação tenha possibilitado que as bananeiras suprissem suas necessidades de $\mathrm{K}$ a partir das reservas potássicas do solo.

Observou-se em campo que a durabilidade das folhas 
TABELA 1 - Análise química do solo da área experimental. Valores médios para toda a área de amostras coletadas na implantação do bananal (setembro de 1997)

\begin{tabular}{|c|c|c|c|c|c|c|c|c|c|c|}
\hline Profundidade & $\mathrm{pH}\left(\mathrm{C} \mathrm{a} \mathrm{C}_{2}\right)$ & M O & $\mathrm{P}_{(\mathrm{resin} a)}$ & $\mathrm{K}$ & $\mathrm{C} \mathrm{a}$ & $\mathrm{Mg}$ & $\mathrm{H}+\mathrm{A} \mathrm{l}$ & S B & $\mathrm{T}$ & $\mathrm{V}$ \\
\hline$-\cdots--\mathbf{c m} \quad-\cdots$ & & $\mathrm{g} \mathrm{d} \mathrm{m}^{-3}$ & $\mathrm{mg} \mathrm{d} \mathrm{m}^{-3}$ & $--\cdot$ & $\cdots-\cdots$ & - $\mathbf{m ~ m}$ & $\mathrm{I}_{\mathrm{c}} \mathrm{d} \mathrm{m}^{-3}$ & & -- & $\%$ \\
\hline $0-20$ & 5,3 & 22 & 46 & 3,4 & 32 & 13 & 34 & 48,4 & 82,4 & 59 \\
\hline $20-40$ & 5,2 & 18 & 20 & 2,9 & 25 & 10 & 32 & 37,9 & 69,9 & 54 \\
\hline
\end{tabular}

TABELA 2 - Resumo da análise de variância do número de folhas na colheita, índice de durabilidade foliar, teores de $\mathrm{N}$ e K e relação $\mathrm{K} / \mathrm{N}$ na folha-índice de bananeira em dois ciclos de cultivo

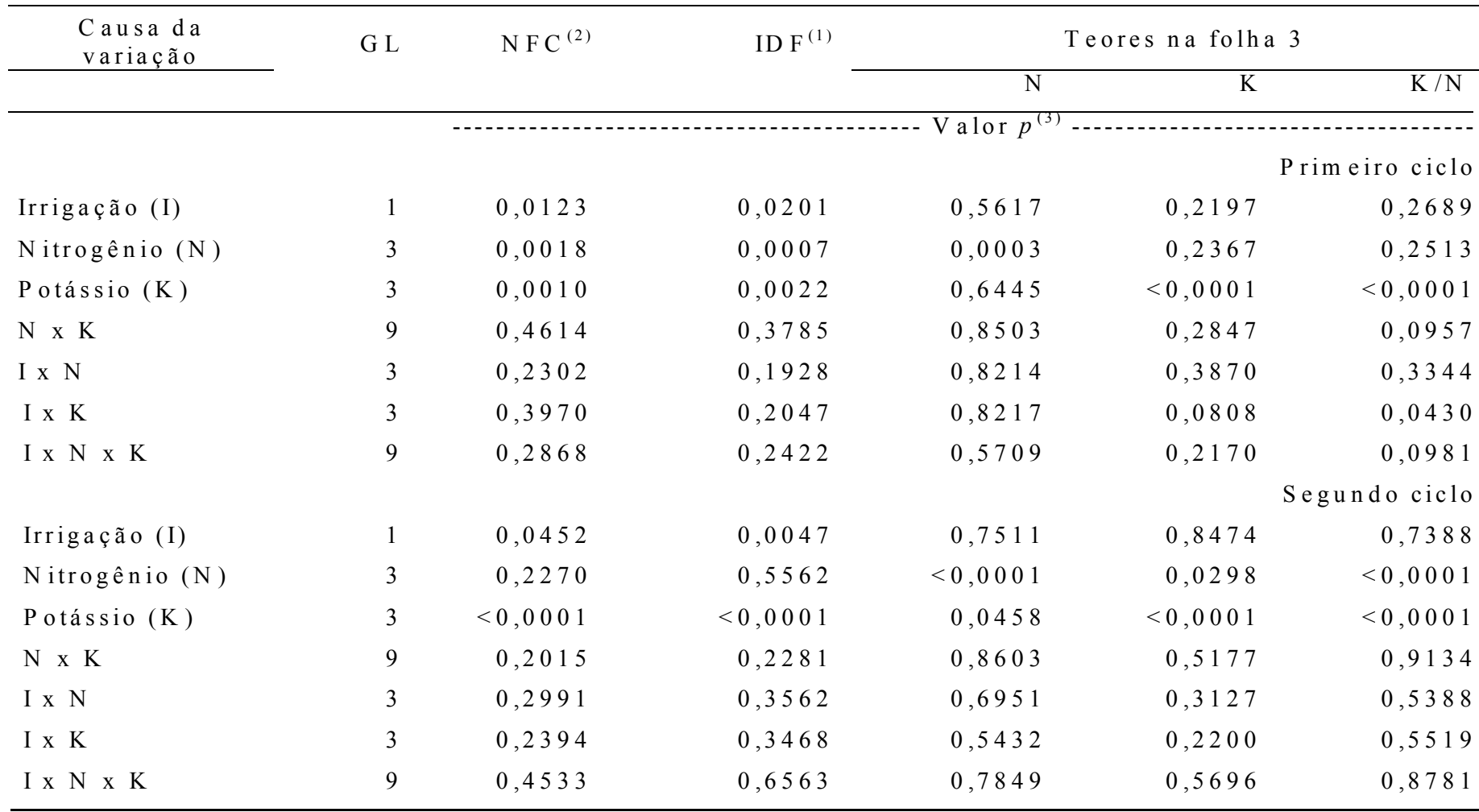

Valores em negrito: $p<0,05$ (F significativo a $5 \%$ de probabilidade); ${ }^{(1)} \mathrm{IDF}=($ Número de folhas na colheita $\div$ número na emissão da inflorescência) $\times 100$; (2) NFC=Número de folhas na colheita; (3) Valor $p$ : probabilidade de erro tipo I associada ao teste $\mathrm{F}$.

TABELA 3 - Médias do número de folhas na colheita, índice de durabilidade foliar, teores de $\mathrm{N}$ e K e relação K/N na folha-índice de bananeira, sob dois regimes hídricos, em dois ciclos de cultivo

\begin{tabular}{|c|c|c|c|c|c|}
\hline \multirow{2}{*}{$\begin{array}{l}\text { Regime } \\
\text { hídrico }\end{array}$} & \multirow[t]{2}{*}{$\operatorname{NFC}^{(1)}$} & \multirow[t]{2}{*}{ ID F $^{(2)}$} & \multicolumn{3}{|c|}{ Teores na folha 3} \\
\hline & & & $\mathrm{N}$ & K & $\mathrm{K} / \mathrm{N}$ \\
\hline & & $\%$ & & -------- & ----------- \\
\hline & & & & & eiro ciclo \\
\hline Sequeiro & $3,6 a^{(3)}$ & 27 a & 25,6 a & 18,5 a & 0,72 a \\
\hline Irrigado & $7,4 \mathrm{~b}$ & $51 \mathrm{~b}$ & 26,0 a & 24,3 a & 0,95 a \\
\hline C.V. (\%) & 5,3 & 7,9 & 7,7 & 39,1 & 49,8 \\
\hline & & & & & undo ciclo \\
\hline Sequeiro & $4,1 \mathrm{a}$ & 32 a & 22,4 a & 23,9 a & $1,09 a$ \\
\hline Irrigado & $7,0 \mathrm{~b}$ & 55 b & 22,8 a & 24,0 a & 1,07 a \\
\hline C.V.(\%) & 14,8 & 1,6 & 19,2 & 4,7 & 19,5 \\
\hline
\end{tabular}

(1) NFC=Número de folhas na colheita; (2) IDF=(número de folhas na colheita $\div$ número na emissão da inflorescência) $\times 100$; ${ }^{(3)}$ Valores num mesmo ciclo de cultivo, seguidos por letras iguais, não diferem entre si (teste $\mathrm{F}, p>0,05$ ). 


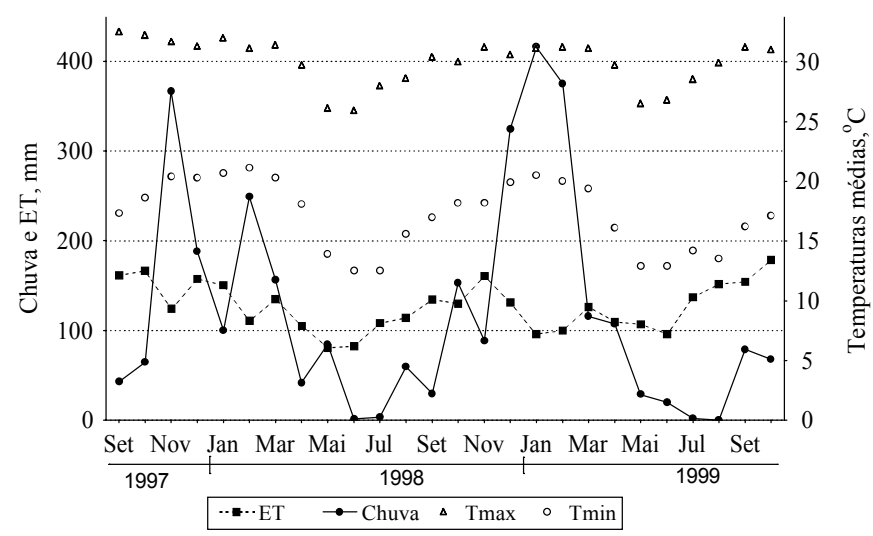

FIGURA 1 - Dados meteorológicos coletados durante a condução do experimento. $\mathrm{ET}=\mathrm{kp}$ ' evaporação no tanque Classe A (total mensal, mm); Chuva = precipitação total mensal $(\mathrm{mm}) ;$ Tmax $=$ média mensal das temperaturas máximas diárias $\left({ }^{\circ} \mathrm{C}\right) ;$ Tmin $=$ média mensal das temperaturas mínimas diárias.

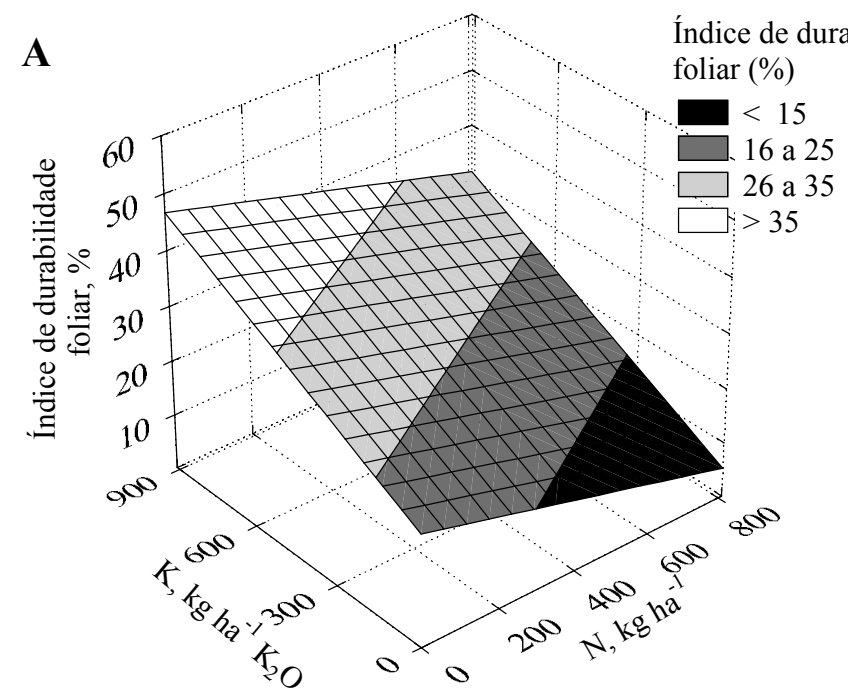

$\mathrm{IDF}_{\text {irir }}=\mathrm{f}(\mathrm{N}$ ou $\mathrm{K})$ n.s. $\quad \overline{\mathrm{Y}}=51$

$\mathrm{IDF}_{\text {seq }}=20,58-1,89 \times 10^{-2} \mathrm{~N}+2,87 \times 10^{-2} \mathrm{~K} \quad \mathrm{R}^{2}=0,67 * *$

B

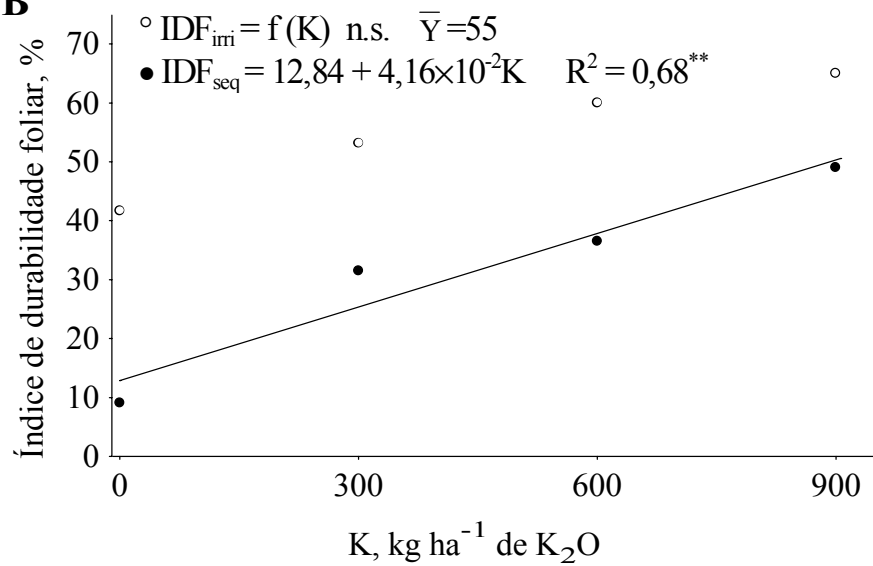

FIGURA 2 - Índice de durabilidade foliar (100 ' número de folhas na colheita,número de folhas na emissão da inflorescência) em função de doses de $\mathrm{N}$ e K, no primeiro ciclo (a), e em função de doses de $\mathrm{K}$, no segundo ciclo (B) de bananeiras.

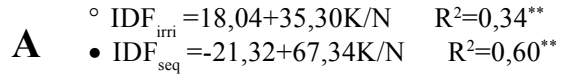
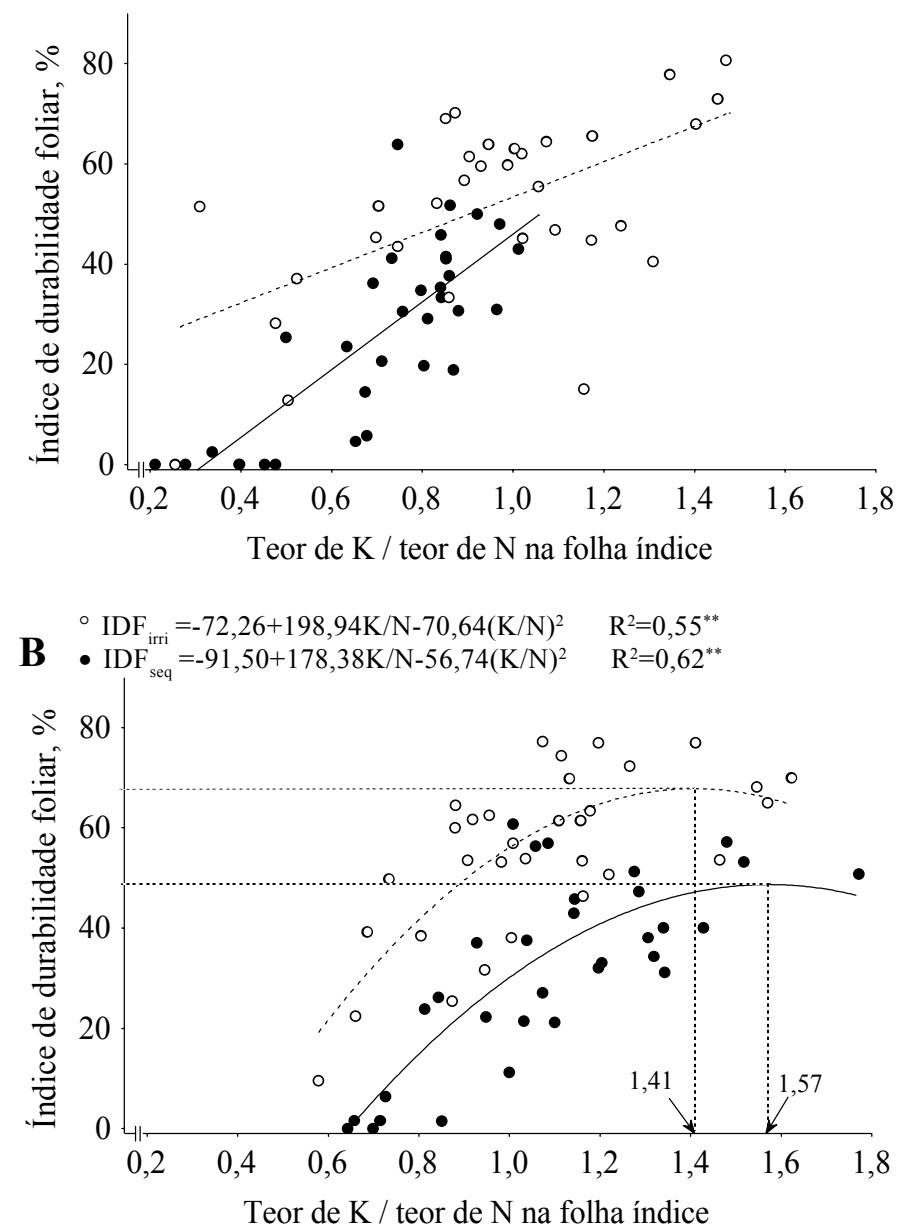

FIGURA 3 - Índice de durabilidade foliar (100'número de folhas na colheita,número de folhas na emissão da inflorescência) em função da razão do teor de K/N, na folha-índice de bananeira, sob dois regimes hídricos, no primeiro (a) e segundo (b) ciclos. Valores assinalados na abscissa estimam as razões $\mathrm{K} / \mathrm{N}$ que maximizaram o índice de durabilidade.

variava em função dos tratamentos de adubação com N e K. O secamento de folhas que ocorria no período entre a emissão da inflorescência e a época de colheita dos cachos, correspondia aproximadamente aos sintomas de deficiência de $\mathrm{K}$ descritos por Martin-Prével \& Charpentier (1964). As plantas não apresentavam alterações significativas de cor ou arquitetura até próximo da época da emissão da inflorescência. A partir desse momento, as folhas mais velhas amareleciam subitamente, necrosando logo em seguida. Essas parcelas quase sempre correspondiam aos tratamentos de doses mais altas de $\mathrm{N}$ e dose zero de $\mathrm{K}$. A partir dessa constatação, buscou-se verificar se havia alguma relação entre o índice de durabilidade foliar e os teores de $\mathrm{K}$ e $\mathrm{N}$ na folhaíndice. Na Figura 3, tem-se o IDF expresso em função da razão entre os teores foliares de K e N. No primeiro ciclo de cultivo, houve um incremento linear na durabilidade das folhas com o aumento da relação K/N foliar. No segundo ciclo, a durabilidade foliar variou numa relação quadrática com a razão K/N. Nesse ciclo, a máxima durabilidade foliar foi obtida com uma razão K/N em torno de 1,6 para sequeiro e de 1,4 sob irrigação. Acredita-se 
que as doses crescentes de $\mathrm{N}$ tenham favorecido o crescimento e a translocação de nutrientes para os frutos. Esse fato, sem o suficiente aporte de $\mathrm{K}$, determinou incremento na velocidade de senescência foliar. Outrossim, o potássio, segundo Beringer \& Trolldenier (1979), atua na regulação osmótica e resistência das plantas ao estresse hídrico, fazendo com que plantas com nutrição adequada desse elemento apresentem níveis mais baixos de ácido abcísico (ABA), fito-hormônio que acelera a senescência foliar.

\section{CONCLUSÕES}

A irrigação possibilitou a manutenção de folhas fotossinteticamente ativas em bananeiras no período entre a emissão da inflorescência e a colheita do cacho.

A adubação potássica favoreceu a manutenção de folhas ativas, especialmente sob sequeiro.

A senescência das folhas em bananeiras, entre a época da emissão da inflorescência e a colheita dos cachos, foi acelerada em condições de desequilíbrio nutricional de $\mathrm{N}$ e K.

\section{AGRADECIMENTOS}

À FAPESP, pelo auxílio financeiro (processo 96/4209-4) e à Dr $^{\mathrm{a}}$. Aline de Holanda Nunes Maia, pesquisadora da EMBRAPA/Meio Ambiente, pelas orientações para análise estatística.

\section{REFERÊNCIAS BIBLIOGRÁFICAS}

BATAGLIA, O.C., FURLANI, A.M.C., TEIXEIRA, J.P.F., FURLANI, P.R., GALLO, J.R. Métodos de análise química de plantas. Campinas: IAC, 1983.48p. (Boletim Técnico, 78).

BERINGER, H.; TROLLDENIER, G. Influence of K nutrition on the response to environmental stress. In: IPI. Potassium research - review and trends. Bern: IPI, 1979. p.189-222.

BOYER, J.S. Water deficits and photosynthesis. In: KOZLOWSKY, T.T. (Ed.) Water deficits and plant growth. New York: Academic Press, 1976. p.153-90.

DELVAUX, B. Soils. In: GOWEN, S. (Ed.) Bananas and plantains. London: Chapman \& Hall, 1995. p.230-57.

FREUND, R.J.; LITTELL, R.C. SAS for linear models: a guide to the ANOVA and GLM procedures. Cary: SAS Institute, 1981. $231 \mathrm{p}$.

HASSELO, H.N. Premature yellowing of Lacatan bananas.
Tropical Agriculture, London, v.38, p.29-34, 1961.

HOLDER, G.D.; GUMBS, F.G. Effects of water supply during floral initiation and differentiation on female flower production by Robusta bananas. Experimental Agriculture, Cambridge, v.18, p.183-93, 1982.

LITTELL, R.C.; MILLIKENN, G.A.; STROUP, W.W.; WOLFINGER, R.D. SAS system for mixed models. Cary: SAS Institute, 1996.

LAHAV, E. Banana nutrition. In: GOWEN, S. (Ed.) Bananas and plantains. London: Chapman \& Hall, 1995. p.258-316.

LAHAV, E. Effect of different amount of potassium on the growth of the banana. Tropical Agriculture, Guildford, v.49, p.321-35, 1972.

MANICA, I.; SIMÃO, S.; SCARDUA, R. Irrigação em sulcos e sua influência no crescimento e produção da planta matriz de banana (Musa cavendishii Lambert) cv. 'Nanicão'. Revista Ceres, Viçosa, v.22, p.88-108, 1975.

MARTIN-PRÉVEL, P. Bananier. In: MARTIN-PRÉVEL, P.; GAGNARD, J.; GAUTIER, P. (Eds.) L'analyse végétale dans le contrôle de l'alimentation des plantes tempérées et tropicales. Paris: Tec\&Doc, 1984. cap.12, p.715-51.

MARTIN-PRÉVEL, P., CHARPENTIER, J.M. Sintomas de carência em seis elementos minerais na bananeira. Fertilité, Paris, v.22, p.15-20, 1964.

MURRAY, D.B. The effect of deficiencies of the major nutrients on growth and leaf analysis of the banana. Tropical Agriculture, Trinidad, v.38, p.123-32, 1960.

MOREIRA, R.S. Banana: teoria e prática de cultivo. 2.ed. São Paulo: Fundação Cargill, 1999. (CD ROM)

RAIJ, B.van, QUAGGIO, J.A. Métodos de análise química de solo para fins de fertilidade. Campinas: IAC, 1983. 31p. (Boletim Técnico, 81)

ROBINSON, J.C.; ANDERSON, T.; ECKSTEIN, K. The influence of functional leaf removal at flower emergence on components of yield and photosynthetic compensation in banana. Journal of Horticultural Science, Ashford, v.67, p.403-10, 1992.

SOUZA, J. S., TORRES FILHO, P. Aspectos socioeconômicos. In: ALVES, S.J. A cultura da banana: aspectos técnicos socioeconômicos e agroindustriais. Brasília: EMBRAPA-SPI/ EMBRAPA-CNPMF, 1997. p.507-524. 